\title{
Multi-way correspondence analysis approach to examine Nobel Prize data from 1901 to 2018
}

\author{
T. Alhuzali ${ }^{a}$, E. J. Beh ${ }^{a}$ and E.Stojanovski ${ }^{a}$ \\ ${ }^{a}$ School of Information and Physical Sciences, University of Newcastle \\ Email: TurkiAbdulrahmanT.Alhuzali@uon.edu.au
}

\begin{abstract}
This study examines Nobel Prize data by studying the association between the nationality of the laureate, the discipline in which the Nobel Prize was awarded, and the gender of the recipient by maintaining the multi-way structure of the data. A three-way contingency table is formed by simultaneously crossclassifying the three categorical variables using a three-way correspondence analysis to assess the association between the three variables. The significance of this study lies in preserving the multivariate associations, which the multiple correspondence analysis approach does not allow. The multi-way correspondence analysis (MWCA) maintains all three-way associations as well as the pair-wise structures between the variables in the case of three variables. The present study consists of 785 individuals from eight developed countries that received a Nobel Prize in the period from 1901 to 2018 (inclusive) - the countries being Canada, France, Germany, Italy, Japan, Russia, British Isles and the United States of America, while the disciplines in which the individuals were awarded the prize include chemistry, physics, physiology or medicine, literature, economics and peace. The results from the MWCA suggest that a strong symmetric association exists between the three variables, in addition, there is a statistically significant association between each pair-wise combination of the variables. The application shows that male physics recipients tend to be from Russia, Japan, and France while female recipients were more likely to be from Japan and France. Furthermore, the analysis highlights that the female medicine recipients are predominantly from the United States of America and the British Isles.
\end{abstract}

Keywords: Multi-way correspondence analysis, symmetric, asymmetric, Nobel Prize 


\section{INTRODUCTION}

The Nobel Prize is an international prestigious award bestowed to eminent scientists who contribute significant works in the fields of science and culture. The first prize was awarded on the fifth anniversary of the death of the Prize's founder, Alfred Nobel, in 1901, and has since been awarded annually to individuals who contribute significant works that benefit fields such as medicine, physiology, physics, chemistry, peace, literature and economics (Morais, 2018). Between 1901 and 2018, a total of 785 prizes were awarded to individuals or teams who contributed significant works that benefited these disciplines.

Since the prize was first awarded in 1901, there have been only a very small proportion of female Nobel laureates compared to males. Given that women have historically occupied considerably fewer academic positions than men, it might then be expected that more Nobel laureates are male than female (Lunnemann, Jensen, \& Jauffred, 2019). Goulden et al. (2009) indicates that female post-doctorals who have either become parents or expect to have children in the future are more likely to abandon research careers, and they are twice as likely as males in the same situations to do so. Nussbaum (2004) argues that the limited number of females seeking higher education in research is one of the potential factors that has led to fewer female Nobel laureates. However, over the past few decades, the proportion of females in scientific careers has increased in all fields of research (Shen, 2013). Females have become more career-oriented and there has been increased involvement of females in science in recent decades (Modgil et al., 2018).

The purpose of this paper is to look more closely at the recipients of the Nobel Prize and examine the association between their Country of nationality, the Discipline in which they received their award, and the Gender of awardee. Analysing categorical data that consists of more than two categorical variables often requires a data visualisation technique that highlights the association between the variables by transforming the multi-way contingency table to a two-way matrix form as in classical multiple correspondence analysis (MCA). In terms of analysing associations between three categorical variables simultaneously, the analysis of a three-way contingency table may be undertaken without having to collapse any of the variables that gives a data structure that is in a two-way form; see for example, Kroonenberg (1989), Carlier \& Kroonenberg (1996), and Beh \& Lombardo (2014, 2019). Examining the structure of three-way contingency tables requires a methodology that visualises the association between the three categorical variables. An example of such an approach is the classical approach to the MCA, which involves coding the multi-way table into a two-way matrix structure. From such coding, MCA does not incorporate the true multivariate association structures between variables but instead reflects only the pairwise association structure that exists. See, for example, Beh and Lombardo (2014, Chapter 11; 2019). This paper will use multi-way correspondence analysis (MWCA) to examine the association between Nobel Prize winner's Country of nationality, the Discipline in which they received their award, and the Gender of awardee.

Section 2.1 describes the data that we are analysing and provides an introduction to MWCA, which is presented in Section 2.2. In Section 3.1, an overview of the data for the Nobel Prize based on the gender of the recipient is provided and an evaluation of the association between the three variables Country, Discipline, and Gender is presented in section 3.2 .

\section{METHOD}

\subsection{Data}

A total of $n=785$ laureates were awarded a Nobel Prize between 1901 and 2018; an overview of the awards granted is described in various detail by the Nobel Foundation (NobelPrize.org, 2019) and Encyclopedia Britannica (Encyclopedia Britannica, 2019). The study consists of three categorical variables; Country of the laureate, Discipline in which the prize was awarded, Gender of the recipient. The Country variable categories comprised eight countries; Germany (DEU), France (FRA), Canada (CAN), Japan (JAP), Italy (ITA), Russia (RUS), the United States of America (USA) and British Isles (BI). Discipline categories comprised: Chemistry (Chm), Economy (Eco), Literature (Lit), Physiology and Medicine (Med), Peace (Pce) and Physics (Phy); and Gender comprised $\mathrm{M}$ and $\mathrm{F}$ for male and female, respectively.

\subsection{Statistical analysis}

MWCA is a valuable visualisation instrument for evaluating the structure of the association between the variables of a three-way contingency table by preserving its cubic format. In correspondence analysis (CA), a common method to decompose the transformed form of a two-way contingency table into component matrices that provide the necessary numerical results needed to graphically summarise the association between the categorical variables is the singular value decomposition (SVD); see Greenacre (1984) and Beh and Lombardo 
(2014). However, because SVD is only suitable for a two-way matrix, a multi-way version of SVD is required to preserve the (hyper-) cube format of the data. In this case, consideration of decomposition methods such as the CANDECOMP/PARAFAC and Tucker's methods are needed; see Tucker (1966), Carroll \& Chang (1970), Harshman (1970), Carlier and Kroonenberg (1996), Kroonenberg (1983, 1989, 2008), and Beh and Lombardo (2014, 2019). Our discussion focus will be on the Tucker3 decomposition approach; a three-way generalisation of SVD used for decomposing a multi-way contingency table (Tucker, 1966). It is assumed that the variables are symmetrically associated such that they are all considered to be predictor variables. In this case, the strength of the association among variables can be measured using Pearson's phi-squared statistic which is typically referred to as the total inertia of the contingency table in the CA literature; see, for example, Carlier \& Kroonenberg (1996).

For a three-way correspondence analysis, consider a $I \times J \times K$ three-way contingency table, denoted as $\boldsymbol{N}$, in which the $(i, j, k)$ th cell entry is denoted by $n_{i j k}$ for $i=1,2, \ldots, I, j=1,2, \ldots$, Jand $k=1,2, \ldots, K$. Consider $n$ as the aggregate of $\boldsymbol{N}$ and $\boldsymbol{P}=\left(p_{i j k}\right)$ the joint relative frequency table associated with $\boldsymbol{N}$, so that, $p_{i j k}=n_{i j k} / n$ and $\sum_{i=1}^{I} \sum_{j=1}^{J} \sum_{k=1}^{K} p_{i j k}=1$. Define $p_{. . k}=\sum_{i=1}^{I} \sum_{j=1}^{J} p_{i j k} ; p_{. j .}=\sum_{i=1}^{I} \sum_{k=1}^{K} p_{i j k} ; p_{i . .}=\sum_{j=1}^{J} \sum_{k=1}^{K} p_{i j k}$ to be the univariate marginal proportions of the $k$ th tube category, $j$ th column, and $i$ th row respectively. Also, denote $p_{i j .}=\sum_{k=1}^{K} p_{i j k} ; p_{i . k}=\sum_{j=1}^{J} p_{i j k} ; p_{. j k}=\sum_{i=1}^{I} p_{i j k}$ to be bivariate marginal proportions. Let $\boldsymbol{I}, \boldsymbol{J}, \boldsymbol{K}$ be the identity matrix of order $I \times I, J \times J$ and $K \times K$, respectively, in the space $\mathrm{R}^{I}, \mathrm{R}^{J}$ and $\mathrm{R}^{K}$, and let $\mathbf{D}_{I}, \mathbf{D}_{J}$ and $\mathbf{D}_{\boldsymbol{K}}$ be a diagonal matrices whose diagonal elements are, respectively, $p_{i . .}, p_{. j .}$ and $p_{. . k}$.

Since the variables are symmetrically associated, Pearson's three-way chi-squared statistic is calculated as

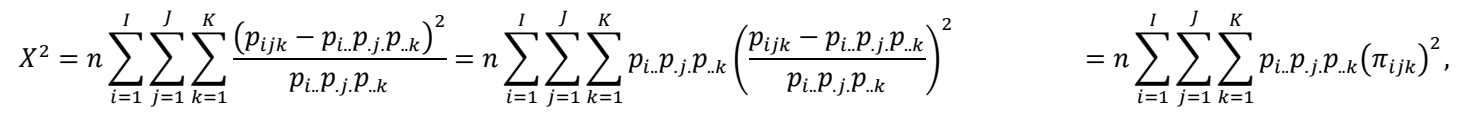

where $\pi_{i j k}=\frac{p_{i j k}}{p_{i . .} p_{. j .} p_{. . k}}-1$ is the $(i, j, k)$ th cell dependency measure. In MWCA the association is quantified using the total inertia $\left(\Phi^{2}=X^{2} / n\right)$ of the three-way contingency table and measures the global deviation of the association between the three categorical variables against what is expected under complete independence where, for the $(i, j, k)$ th cell, $p_{i j k}=p_{i . .} p_{. j .} p_{. . k}$. The statistic $\Phi^{2}$ defined by dividing Equation (1) by $n$ can be partitioned as $\Phi^{2}=\Phi_{I J}^{2}+\Phi^{2}{ }_{I K}+\Phi_{J K}^{2}+\Phi^{2}{ }_{I J K}$. Hence

$$
X^{2}=X_{I J}^{2}+X_{I K}^{2}+X_{J K}^{2}+X_{I J K}^{2},
$$

where each term is a chi-squared random variable, so that $X^{2}$ has $(d f)=(I-1)(J-1)+(I-1)(K-1)+$ $(J-1)(K-1)+(I-1)(J-1)(K-1)$ degrees of freedom. The initial three terms on the right-hand side of Equation (2) represent the three bivariate association measures of the $X^{2}$ test while the last term represents the trivariate association measure of $X^{2}$ between the three variables; see, Carlier and Kroonenberg (1996), Lombardo, Takane and Beh (2019) and Loisel and Takane (2016).

Using the Tucker3 decomposition, MWCA of a three-way contingency table can be performed, such that

$$
\pi_{i j k}=\sum_{p=1}^{P} \sum_{q=1}^{Q} \sum_{r=1}^{R} a_{i p} b_{j q} c_{k r} \lambda_{p q r}+e_{i j k}
$$

where $P, Q$ and $R(P \leq I, Q \leq J, R \leq K)$ are the fixed number of the components required for visualising the rows, columns, and tubes, respectively. Here, $a_{i p}$ is the $i$ th row score along the $p$ th dimension of a correspondence plot for $p=1,2, \ldots, P \leq I$, and $i=1,2, \ldots, I$. Similarly, $b_{j q}$ is the $j$ th column score along the $q$ th dimension belonging to a correspondence plot for $q=1,2, \ldots, Q \leq J$ and $j=1,2, \ldots, J$. Finally, $c_{k r}$ is the $k$ th tube score along the $r$ th dimension belonging to a correspondence plot for $r=1,2, \ldots, R \leq K$ and $k=$ $1,2, \ldots, K$. The quantity $\lambda_{p q r}$ is the $(p, q, r)$ th element of the core array which can be viewed as a three-way generalisation of the singular value obtains from SVD. The term $e_{i j k}$ represents the error of approximation, and when $R=K, Q=J$, and $P=I$ then all $e_{i j k}=0$. The total inertia can be denoted in relation to $\lambda_{p q r}$ as

$$
\Phi^{2}=\sum_{p=1}^{P} \sum_{q=1}^{Q} \sum_{r=1}^{R} \lambda_{p q r}^{2}
$$

To determine the appropriate $P, Q$ and $R, e_{i j k}=\pi_{i j k}-\hat{\pi}_{i j k}$ is minimized, where

$$
\hat{\pi}_{i j k}=\sum_{p=1}^{P} \sum_{q=1}^{Q} \sum_{r=1}^{R} a_{i p} b_{j q} c_{k r} \lambda_{p q r}
$$


is the expected value of $\pi_{i j k}$ for some value of $P, Q$ and $R$. Their choice is made by maximising $\left(\sum_{i j k} \hat{\pi}_{i j k}{ }^{2}\right) / \Phi^{2}$ while ensuring $P, Q$ and $R$ provide interpretable components.

Let $\boldsymbol{a}_{p}, \boldsymbol{b}_{q}$, and $\boldsymbol{c}_{r}$ be vectors whose elements are $a_{i p}, b_{j q}$, and $c_{k r}$, respectively. These three vectors are orthonormal with weight matrix $\mathbf{D}_{I}, \mathbf{D}_{J}$ and $\mathbf{D}_{K}$ in their respective spaces $\mathrm{R}^{I}, \mathrm{R}^{J}$ and $\mathrm{R}^{K}$, respectively, and are calculated using a weighted alternating least-squares algorithm; see also, Carlier \& Kroonenberg (1996) and Beh \& Lombardo (2014). To select which dimensions of the column-tube space can be considered, we examine the percentage of the total inertia explained by these components.

To graphically summarize the symmetric association among the variables of the three-way contingency table, we consider the column-tube interactive biplot described in Beh \& Lombardo (2014, Section 11.9.2). Such a biplot specifies an array of principal coordinates for the column and tube variables assuming that there is an association between them.

In the construction of a column-tube interactive biplot, definition of the $i$ th row standard coordinate as well as the $(j, k)$ th column-tube principal coordinate along the $p$ th dimension of the biplot becomes,

$$
\hat{f}_{i p}=a_{i p} \quad(6) \quad \hat{g}_{j k p}=\sum_{q=1}^{Q} \sum_{r=1}^{R} b_{j q} \lambda_{p q r} c_{k r},
$$

respectively, for $p=1,2, \ldots, P$. Thus, this biplot has an optimal low-dimensional space that consists of $P$ dimensions.

To graphically represent the association between the three variables, the $(i, j, k)$ th Pearson residual can be expressed by the inner product of Equations (6) and (7) such that

$$
\pi_{i j k}-1=\sum_{p=1}^{P} \hat{f}_{i p} \hat{g}_{j k p} .
$$

This shows shows that an association between a column-tube pair and a row exists if the position of the principal coordinate of $(j, k)$ th column-tube is close to the projection of the $i$ th row standard coordinate from the origin; see Carlier \& Kroonenberg (1996) and Beh \& Lombardo (2014, Chapter 11).

\section{RESULTS}

\subsection{An overview of Nobel Prize data}

Table 1 is the $8 \times 6 \times 2$ contingency table based on the cross-classification of the 785 Nobel Prize recipients according to the categorical variables Country, Discipline, and Gender. From Table 1, 742 (94.5\%) of the recipients were male and only $43(5.5 \%)$ were female.

\begin{tabular}{|c|c|c|c|c|c|c|c|c|}
\hline & & \multicolumn{6}{|c|}{ Discipline } & \multirow[b]{2}{*}{ Total } \\
\hline & & $\mathrm{Chm}$ & Eco & Lit & Med & Pce & Phy & \\
\hline \multirow{9}{*}{ Male } & Canada & 6 & 4 & 2 & 6 & 1 & 4 & 23 \\
\hline & France & 13 & 3 & 16 & 12 & 1 & 13 & 58 \\
\hline & Germany & 30 & 1 & 8 & 26 & 7 & 33 & 105 \\
\hline & Italy & 1 & 1 & 5 & 5 & 1 & 5 & 18 \\
\hline & Japan & 7 & 0 & 3 & 5 & 1 & 11 & 27 \\
\hline & Russia & 2 & 2 & 4 & 2 & 3 & 12 & 25 \\
\hline & British Isles & 31 & 11 & 10 & 35 & 11 & 28 & 126 \\
\hline & USA & 77 & 60 & 11 & 95 & 18 & 99 & 360 \\
\hline & Total & 167 & 82 & 59 & 186 & 43 & 205 & 742 \\
\hline \multirow{7}{*}{ Female } & Canada & 0 & 0 & 0 & 0 & 0 & 1 & 1 \\
\hline & France & 2 & 0 & 0 & 1 & 11 & 1 & 15 \\
\hline & Germany & 0 & 0 & 2 & 0 & 0 & 1 & 3 \\
\hline & Italy & 0 & 0 & 1 & 1 & 0 & 0 & 2 \\
\hline & British Isles & 1 & 0 & 1 & 0 & 4 & 0 & 6 \\
\hline & $\begin{array}{c}\text { United States of } \\
\text { America }\end{array}$ & 1 & 1 & 2 & 8 & 3 & 1 & 16 \\
\hline & Total & 4 & 1 & 6 & 10 & 18 & 4 & 43 \\
\hline
\end{tabular}

Table 1. A three-way contingency table based on the cross-classification of Country and Discipline based on Gender of the recipient

For males, Canada and France appear to be relatively consistent with each other in terms of the proportion of economics and peace awards; while chemistry, literature, medicine, and physics appear dominated by French laureates. Germany and the British Isles also appear relatively consistent with each other in terms of their male dominance across most disciplines, except for economics, with the British Isles having received 13\% of 
economics Nobel prizes, higher than Germany. Japan and Russia also obtained very similar results among males, and dominate in physics, with Japan outperforming Russia in chemistry more than threefold. The United States of America is the leading country with male Nobel Prize laureates dominating the majority of disciplines in chemistry $(46 \%)$, economics $(73 \%)$, medicine $(51 \%)$, and physics $(48 \%)$ respectively. However, the USA appears to be consistent with Germany, and the British Isles for the proportion of male laureates in literature. While there are more males than females awarded Nobel Prizes, female recipients in France received approximately $35 \%$ of all female prizes across all of the considered countries. Furthermore, women in the USA have shown exceptional dominance in Medicine, relative to other countries, claiming $80 \%$ of all medicine prizes awarded to females.

For MCA, the three-way association structure is reduced to investigating multiple pair-wise association structures; see, for example, Beh \& Lombardo (2014). MWCA applied to Table 1, however, preserves the multi-way structure of the data, compared with MCA that only involves coding the multi-way table into twoway matrix structures. This therefore allows for an investigation of the association between the three variables simultaneously instead of reducing the dimensionality and only investigating multiple pair-wise association structures as is done in MCA.

\subsection{Three-way correspondence analysis}

Since we consider the three variables of Table 1 as being symmetrically associated, Pearson's chi-squared statistic is partitioned in the manner given by Equation (2) so that a three-way and each pairwise association structure between variables can be studied; such a partition is summarised in Table 2 . The total value of the Pearson's chi-squared statistic for three-way association $\left(X^{2}=536\right)$ shows the existence of a statistically significant association between variables $(p<0.001)$. This implies a strong symmetric association between Country, Discipline and Gender. To examine the nature of this association we consider these terms: $X^{2}{ }_{I J}=$ 107.02, $X_{I K}^{2}=38.73, X_{J K}^{2}=81.16$, and $X_{I J K}^{2}=309.13$. The association between Country and Discipline is statistically significant $(p<0.001)$; see, Alhuzali, Stojanovski \& Beh, (2019), with this association justifying $20 \%$ of the total association present in Table 1 . The two-way association between Discipline and Gender $(p<0.001)$ represents a statistically significant association between the Gender of the Nobel Prize recipient and Discipline, with this association accounting for $15 \%$ of the total association existing between the variables depicted in Table 1. Furthermore, a statistically significant association $(p<0.001)$ is also present between Gender of the Nobel Prize and Country, while this marginal term explains just 7\% of the total inertia.

Table 2. Partitioning of the Pearson's chi-squared statistic for the Nobel Prize data in Table 1

\begin{tabular}{cccccc}
\hline & $\boldsymbol{X}^{2}{ }_{\boldsymbol{I J}}$ & $\boldsymbol{X}^{\mathbf{2}}{ }_{\boldsymbol{I K}}$ & $\boldsymbol{X}_{\boldsymbol{J} \boldsymbol{K}}$ & $\boldsymbol{X}^{\mathbf{2}}{ }_{\text {res }}$ & $\boldsymbol{X}^{\mathbf{2}}{ }_{\text {tot }}$ \\
\hline Index & 107.016 & 38.731 & 81.162 & 309.130 & 536.038 \\
\% Explained inertia & 19.964 & 7.225 & 15.141 & 57.669 & 100.000 \\
df & 35.000 & 7.000 & 5.000 & 35.000 & 82.000 \\
$p$-Value & $<0.001$ & $<0.001$ & $<0.001$ & $<0.001$ & $<0.001$ \\
\hline
\end{tabular}

Table 3 reports four combinations of $P, Q$, and $R$ of the three-way correspondence analysis, the explained inertia value and the percentage of $\Phi^{2}$ that it accounts for. It is noted that $87 \%$ of the total inertia is accounted for when $P=3, Q=3, R=2$ is chosen. However, $85 \%$ of the total inertia is mirrored when using only the first two rows, columns and tube components so choosing $P=Q=R=2$ is considered the superior choice since it explains the highest percentage of the association between variables under the existence of fewest components. The $a_{i p}, b_{j q}, c_{k r}$, and $\lambda_{p q r}$ values can be determined by setting $P=Q=R=2$ and measured using the CA3variants R package of Lombardo, Beh, \& Van de Velden (2020). Table 4 summarises the core array elements, $\lambda_{p q r}$ for $P=Q=R=2$. Components of the first mode are denoted by $p 1$ and $p 2$, the second and third mode components by $q 1, q 2$ and $r 1, r 2$ respectively. The value $\left(\lambda_{111}=-0.7089\right)$; the mixture of the first component of the first mode, $p 1$, the first component of the second mode, $q 1$, and the first component of the third mode, $r 1$, gives a statistically significant negative association, indicating an inverse relationship between the $p 1$ dimension and the combined $q 1 r 1$ component. Similarly, $\left(\lambda_{222}=0.2290\right)$ is a significant positive value, indicating a direct relationship between the second component, $p 2$, of the first mode and the interaction of the second component of the second and third mode, $q 2 r 2$. To summarise the visualisation of symmetrical association between the variables of a three-way contingency table, the columntube interactive biplot that defines a collection of principal coordinates for the column and tube variables, supposing a present interaction between them, can be used; see, Carlier and Kroonenberg (1996), Lombardo et al., (1996) and Kroonenberg (2008). 
Alhuzali et al., Multi-way correspondence analysis approach to examine Nobel Prize

Table 3. Three-way CA: Model size and explained inertia of three possible models

\begin{tabular}{cccc}
\hline & Model size & Sum of components & Explained inertia \\
\cline { 1 - 2 } & $\boldsymbol{P} \times \boldsymbol{Q} \times \boldsymbol{R}$ & & \\
\hline 1 & $8 \times 6 \times 2$ & 16 & $100 \%$ \\
2 & $3 \times 3 \times 2$ & 8 & $87 \%$ \\
3 & $2 \times 2 \times 2$ & 6 & $85 \%$ \\
4 & $2 \times 2 \times 1$ & 5 & $76 \%$ \\
\hline
\end{tabular}

Table 4. The core array elements, $\lambda_{p q r}$ from the Tucker3 of $\pi_{i j k}$ of Table 1

\begin{tabular}{|c|c|c|c|}
\hline & $q 1$ & $q 2$ & \\
\hline$p 1$ & -0.7089 & -0.0097 & \multirow[b]{2}{*}{$r 1$} \\
\hline$p 2$ & 0.0506 & 0.0296 & \\
\hline \multicolumn{2}{|r|}{$q 1$} & $q 2$ & \\
\hline$p 1$ & 0.0045 & 0.1586 & \multirow[b]{2}{*}{$r 2$} \\
\hline$p 2$ & -0.0397 & 0.2290 & \\
\hline
\end{tabular}

Since $\left(\Phi_{\text {tot }}^{2}=536.038 / 785=0.6829\right)$, the phi-squared statistic, that is the sum-of-squares of the elements in Table 4 , is $(0.5848 / 0.6829=0.85)$, indicating that $85 \%$ of total inertia is interpreted, which is a substantial percentage contribution by the three components to the total variation through the first two dimensions, as depicted in Figure 1. Based on Figure 1, PceF (the interaction of categories "peace" and "female") appears at the far right on the plot, hence making a significant contribution to the first dimension; while LitM (the interaction of categories "literature" and "male") makes a strong contribution along the two dimensions. Furthermore, categories France and British Isles have the most impactful contributions to the association, relative to the remaining countries. We observe that column-tubes PceF, LitM, and EcoM (interaction of categories "economic" and "male") contribute the most to the association due to their distance from the origin. However, the interaction between male recipients and disciplines of physics and chemistry, is not very strong and so they do not contribute significantly to describing the association structure between the three variables due to their closeness to the origin. Similarly, for female recipients in the disciplines of medicine and physics.

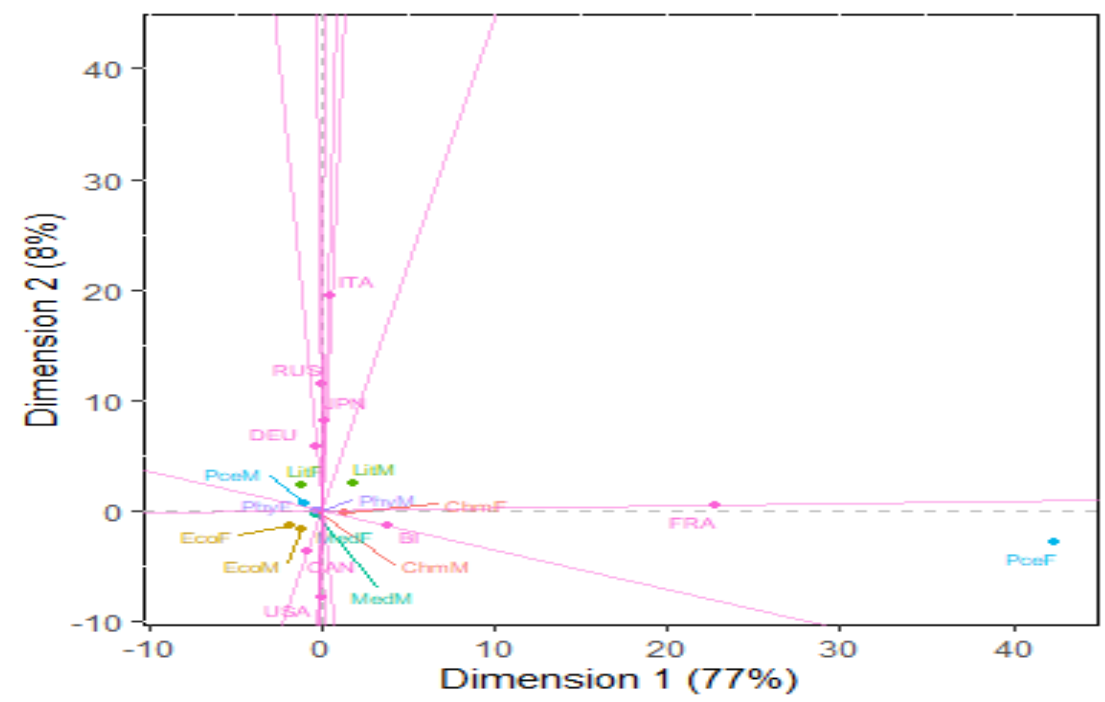

Figure 1. Column-tube interactive biplot of three-way symmetrical correspondence analysis

\section{DISCUSSION}

Three-way correspondence analysis was used to assess the association between Country of the nominated individual (or team), Discipline in which Nobel Prize was awarded, and Gender of recipient. Using the CA3variants $\mathrm{R}$ package, the column-tube interactive biplot was used to graphically represent row points and column-tube points in a single graph to help with gaining an informative interpretation association between the variables. The results from MWCA show the bivariate/trivariate association measurements between Country, Discipline, and Gender. The results also describe the association between categories of these 
variables. Findings indicate male physics winners as more likely from Russia, Japan, and France, while female recipients were more likely from Japan and France. Females in the USA and the British Isles have solid contributions in medicine, compared with other countries. The study variables in this analysis were assumed symmetrically associated and were treated as predictor variables. Due to questions on the impact of establishing the gender or country of origin of a Nobel laureate on their discipline, scenarios in which the variables are asymmetrically associated can also be considered as there can be a dependency between variables in future studies.

\section{REFERENCES}

Alhuzali, T., Stojanovski, E., \& Beh, E.J. (2019). Correspondence analysis approach to examine the Nobel Prize. In Elsawah, S. (ed.) MODSIM2019, 23rd International Congress on Modelling and Simulation. Modelling and Simulation Society of Australia and New Zealand, pp. 284-290.

Beh, E. J., \& Lombardo, R. (2014). Correspondence Analysis: Theory, Practice and New Strategies: Wiley.

Beh, E. J., \& Lombardo, R. (2019). Multiple and multiway correspondence analysis. Wiley Interdisciplinary Reviews: Computational Statistics, 11(5), e1464.

Carlier, A., \& Kroonenberg, P. M. (1996). Decompositions and biplots in three-way correspondence analysis. Psychometrika, 61, 355-373.

Carroll, J. D., \& Chang, J. J. (1970). Analysis of individual differences in multi-dimensional scaling via an nway generalisation of "Eckart - Young" decomposition. Psychometrika, 35, 283-319.

Encyclopedia Britannica. (2019). Nobel Prize. Available at: https://www.britannica.com/topic/Nobel-Prize (Accessed 25 November, 2020].

Goulden, M., Frasch, K., \& Mason, M. A. (2009). Staying competitive: Patching America's leaky pipeline in the sciences. Berkeley, CA: Center for American Progress. Retrieved 6 September 2021, from https://www.americanprogress.org/issues/women/reports/2009/11/10/6979/staying-competitive/

Greenacre, M., \& Blasius, J. (eds) (2006). Multiple Correspondence Analysis and Related Methods: Chapman \& Hall/CRC Press.

Greenacre, M. J. (1984). Theory and Applications of Correspondence Analysis. Academic Press, London.

Harshman, R. A. (1970). Foundations of the PARAFAC procedure: Models and conditions for an "explanatory" multimodal factor analysis. UCLA Working Papers in Phonetics, 16, 1-84.

Kroonenberg, P.M. (1983) Three Mode Principal Component Analysis. DSWO Press, Leiden.

Kroonenberg, P.M. (1989) Singular value decomposition of interactions in three-way contingency tables. In Multiway Data Analysis (eds. R. Coppi and S. Bolasco), pp. 169--184. Elsevier.

Kroonenberg, P.M. (2008) Applied Multiway Data Analysis. John Wiley \& Sons, Inc., New York.

Loisel, S., \& Takane, Y. (2016). Partitions of Pearson's chi-square statistic for frequency tables: A comprehensive account. Computational Statistics, 31(4), 1429-1452.

Lombardo, R., Beh, E. J., \& Van de Velden, M. (2020). CA3variants (Version 3.0) R package: Three-Way Correspondence Analysis Variants. Available at https://cran.rproject.org/web/packages/CA3variants/index.html

Lombardo, R. Carlier, A., and D'Ambra, L. (1996) Nonsymmetric correspondence analysis for threeway contingency tables. Methodologica, 4, 59--80.

Lombardo, R., Takane, Y., \& Beh, E. J. (2019). Familywise decompositions of Pearson's chi-square statistic in the analysis of contingency tables. Advances in Data Analysis and Classification, 14, 629-649.

Lunnemann, P., Jensen, M. H., \& Jauffred, L. (2019). Gender bias in Nobel prizes. Palgrave Communications, 5(1), 1-4.

Modgil, S., Gill, R., Sharma, V. L., Velassery, S., \& Anand, A. (2018). Nobel nominations in science: constraints of the fairer sex. Annals of neurosciences, 25(2), 63-79.

Morais, F. (2018). Vision and the Nobel Prize. Arquivos Brasileiros de ofTalmologia, 81(2), $161-165$.

NobelPrize.org. (2019). All Nobel Prizes. Available at: https://www.nobelprize.org/prizes/lists/all-nobelprizes/ (Accessed 25 November, 2020).

Nussbaum, M. C. (2004). Women's education: A global challenge. Signs: Journal of Women in Culture and Society, 29(2), 325-355.

Shen, H. (2013). Mind the gender gap. Nature, 495(7439), 22

Tucker, L. R. (1966). Some mathematical notes on three-mode factor analysis. Psychometrika, 31(3), 279311. 